Document downloaded from:

http://hdl.handle.net/10251/86337

This paper must be cited as:

Puga Vaca, A. (2016). Light-Promoted Hydrogenation of Carbon Dioxide ¿An Overview. Topics in Catalysis. 59(15-16):1268-1278. doi:10.1007/s11244-016-0658-z.

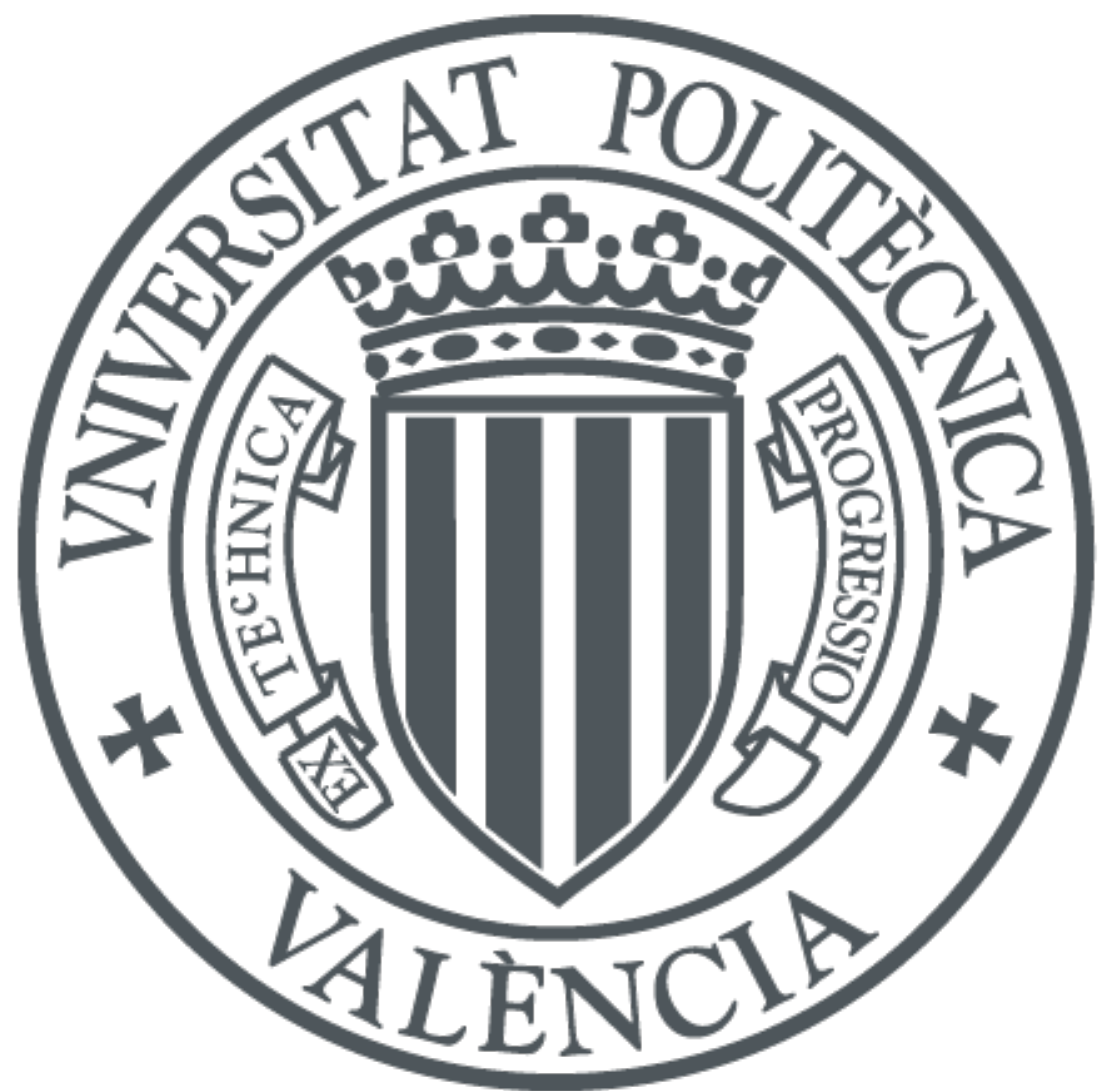

The final publication is available at

http://doi.org/10.1007/s11244-016-0658-z

Copyright Springer Verlag (Germany)

Additional Information 


\title{
Light-Promoted Hydrogenation of Carbon Dioxide-An Overview
}

\author{
Alberto V. Puga \\ a.puga@csic.es
}

Instituto Universitario de Tecnología Química CSIC-UPV, Universitat Politècnica de València, Av. De los Naranjos, s/n, 46022 Valencia, Spain

\begin{abstract}
Hydrogenation of carbon dioxide is considered as a viable strategy to generate fuels while closing the carbon cycle (heavily disrupted by the abuse in the exploitation of fossil resources) and reducing greenhouse gas emissions. The process can be performed by heat-powered catalytic processes, albeit conversion and selectivity tend to be reduced at increasing temperatures owing to thermodynamic constraints. Recent investigations, as summarised in this overview, have proven that light activation is a distinct possibility for the promotion of $\mathrm{CO}_{2}$ hydrogenation to fuels. This effect is particularly beneficial in methanation processes, which can be enhanced under simulated solar irradiation using materials based on metallic nanoparticles as catalysts. The use of nickel, ruthenium and rhodium has led to substantial efficiencies. Lightpromoted processes entail performances on a par with (or even superior to) those of thermallyinduced, industrially-relevant, commercial technologies.
\end{abstract}

Keywords: carbon dioxide, solar fuels, photocatalysis, photothermal effect, methanol, methane.

\section{Introduction}

Recycling carbon dioxide into fuels is considered today as a compelling strategy to compensate for the greenhouse effects caused by increasing emissions [1-2]. The use of renewable energy is the most desirable option to power such process in order to establish pathways for sustainable development for the mid-term future [3]. Among the different known technologies to transform $\mathrm{CO}_{2}$ into hydrocarbon fuels, catalytic hydrogenation is the most mature approach [1-5]. Obviously, the main challenge regarding this is to ensure that the hydrogen needed as the reducing agent is produced from renewable resources and energy $[1,3,6]$, as opposed to the currently dominating pathways relying on fossil fuels.

Catalytic reactions between carbon dioxide and hydrogen can give rise to several transformations (see Table 1), chiefly CO generation via reverse water-gas shift (equation 1), methanol synthesis (equation 2), and methanation (equation 3). 
Table 1 Main reactions involving $\mathrm{CO}_{2}$ and $\mathrm{H}_{2}$ and related changes in thermodynamic potentials at standard conditions

\begin{tabular}{|c|c|c|c|}
\hline equation \# & reaction & $\triangle G^{0} / \mathrm{kJ} \mathrm{mol}^{-1}$ & $\triangle H^{0} / \mathrm{kJ} \mathrm{mol}{ }^{-1}$ \\
\hline 1 & $\mathrm{CO}_{2}(g)+\mathrm{H}_{2}(g) \leftrightarrows \mathrm{H}_{2} \mathrm{O}(g)+\mathrm{CO}(g)$ & 28.6 & 41.2 \\
\hline 2 & $\mathrm{CO}_{2}(g)+3 \mathrm{H}_{2}(g) \leftrightarrows \mathrm{CH}_{3} \mathrm{OH}(g)+\mathrm{H}_{2} \mathrm{O}(g)$ & 3.5 & -53.3 \\
\hline 3 & $\mathrm{CO}_{2}(g)+4 \mathrm{H}_{2}(g) \leftrightarrows \mathrm{CH}_{4}(g)+2 \mathrm{H}_{2} \mathrm{O}(g)$ & -113.6 & -165.0 \\
\hline
\end{tabular}

Reverse water-gas shift is an up-hill (under standard conditions) and endothermic reaction, which is thus favoured at high temperatures. On the other hand, $\mathrm{CO}_{2}$ hydrogenation processes yielding methanol or methane are exothermic, and therefore, conversions tend to diminish at increasing temperatures. These reversible reactions take place simultaneously and a particular system may evolve toward near-equilibrium situations, especially at high temperatures $\left(>300{ }^{\circ} \mathrm{C}\right.$ ), whereby enrichments in $\mathrm{CO}$ have been theoretically predicted and experimentally proven [7] (see Fig. 1). Hydrogenation reactions leading to fuel substances are in contrast favoured at lower temperatures and high pressures $[5,7]$. However, activation of the formidably stable carbon dioxide substrate is challenging due to kinetic barriers, and the design of appropriate catalytic systems that enable the attainment of chemical equilibrium is crucial for the success of $\mathrm{CO}_{2}$-to-fuel technologies. Another point to note is that, in practice, local hot spots can be observed due to heat release as the exothermic hydrogenation reactions proceed [5].

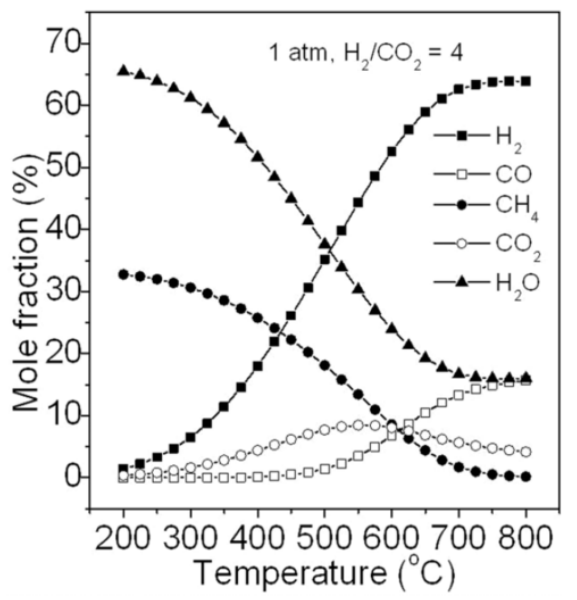

Fig. 1 Calculated product distribution of a typical $\mathrm{CO}_{2}$ methanation feed at equilibrium as a function of temperature. Reproduced from reference [7] (ㅇ The Royal Society of Chemistry, 2012).

Hydrogenation of $\mathrm{CO}_{2}$ can result in the formation of several products, among which, methane (synthetic natural gas) can be obtained at high yields [4-5], whereas other condensable (liquid) fuels such as higher hydrocarbons or methanol are preferred due to their easier storage and transport $[1,4,8]$. These-latter routes are often limited by low selectivity and narrow temperature ranges of operation, respectively and low selectivity. Irrespective of the final product, the reverse water-gas shift reaction leading to $\mathrm{CO}$ (equation 1) has been suggested as 
the initial $\mathrm{CO}_{2}$ conversion step [4,7]. Thus, active catalysts for the hydrogenation of carbon dioxide should also perform satisfactorily for $\mathrm{CO}$ as the substrate, especially regarding resistance to poisoning.

Despite the notable level of advancements in the hydrogenation of carbon dioxide into fuels, several matters of concern still remain. The cost of separation of $\mathrm{CO}_{2}$ from other substances in real streams such as flue gases is one the main problem [9-10], albeit the corresponding technologies are well developed. Furthermore, the use of fossil resources should be minimised by employing renewable energy both to supply hydrogen and to activate the conversion of $\mathrm{CO}_{2}$ into carbon-based fuels $[1,3]$. Regarding the latter topic, harnessing solar energy to power the related processes by means of photocatalysis is attracting significant attention [11-15]. In the context of $\mathrm{CO}_{2}$ hydrogenation, process improvements may be prompted by the use of light [4, 13, 16-17]. Herein, a general overview of research activities on the topic is provided, with special focus on light-promoted productivity enhancements.

\section{Catalytic Hydrogenation of $\mathrm{CO}_{2}$ for the Production of Fuels}

The reduction of carbon dioxide by hydrogen can be activated at moderate temperatures and pressures using a range of catalysts, generally based on metal nanoparticles on oxide supports. Depending on the materials used and on the operating conditions, product distributions can be tuned towards specific outcomes including carbon monoxide, methane, higher hydrocarbons, methanol, dimethyl ether, higher alcohols, or formic species [4]. Most frequently, fixed bed reactors are used for the hydrogenation of $\mathrm{CO}_{2}$. Typical temperatures for methanation processes range between 200 and $400{ }^{\circ} \mathrm{C}$, yet the effect of exothermicity has been shown to cause increases above $600{ }^{\circ} \mathrm{C}$ [5]. The production of highly reduced products (e.g. methanol or methane) is favoured at high pressures, since the corresponding reactions are volume-reducing reversible processes [7], as stated above (see equations 2 and 3 in Table 1). In practice, laboratory testing at atmospheric pressure (for convenience) can provides satisfactory results.

Ruthenium has been claimed as the most efficient metal for $\mathrm{CO}_{2}$ methanation, followed by nickel, iron and cobalt [4-5], whereas iron or mixed cobalt-iron catalysts, reminiscent of Fischer-Tropsch processes, have been used for the production of heavier hydrocarbons [4]. Highly selective methanation of $\mathrm{CO}_{2}$ has been reported to take place at moderate to high conversions (40-90\%) at temperatures above $250{ }^{\circ} \mathrm{C}$ [5] (see Table 2). Consistent with these results, situations near chemical equilibrium can be achieved on $\mathrm{M} / \mathrm{Al}_{2} \mathrm{O}_{3}(\mathrm{M}=\mathrm{Ni}$ [18-19] or $\mathrm{Ru}$ [19]) only at temperatures above $400{ }^{\circ} \mathrm{C}$, although unfortunately, conversions and selectivities to methane decline under such conditions. As mentioned above, lower temperatures are more favourable, and in this regard, striking improvements (100\% conversion, $100 \%$ selectivity to 
$\mathrm{CH}_{4}, 180^{\circ} \mathrm{C}$ ) by using $\mathrm{TiO}_{2}$-supported ruthenium catalysts prepared by a sputtering method have been claimed [20]. Nickel catalysts have also been optimised for performance at moderate temperatures ( $87 \%$ conversion, $99 \%$ selectivity to $\mathrm{CH}_{4}, 250-350{ }^{\circ} \mathrm{C}$ ) by introducing magnesium into oxide solid solution nanoparticles and further encapsulation with silica shells [21]. Selectivity shifts toward preferential $\mathrm{CO}$ formation (with minor formation of $\mathrm{C}_{2}$ products) has been observed by using catalysts based on cobalt nanoparticles supported on mesoporous silica [22], whereas the use of $\mathrm{Ni}_{\text {core }}-\mathrm{Co}_{\text {shell }}$ analogues also leads to the formation of formaldehyde and methanol [23].

Table 2 Selected recent data on catalytic $\mathrm{CO}_{2}$ hydrogenation to methane or methanol

\begin{tabular}{|c|c|c|c|c|c|c|c|c|c|}
\hline \multirow[b]{2}{*}{ catalyst } & \multirow[b]{2}{*}{ feed composition } & \multirow[b]{2}{*}{$\mathrm{P} / \mathrm{bar}$} & \multirow[b]{2}{*}{$\mathrm{T} /{ }^{\circ} \mathrm{C}$} & \multirow[b]{2}{*}{$\begin{array}{r}\chi\left(\mathrm{CO}_{2}\right) / \\
\%\end{array}$} & \multirow[b]{2}{*}{$\begin{array}{r}S\left(\mathrm{CH}_{4}\right) / \\
\%\end{array}$} & \multicolumn{3}{|c|}{$\begin{array}{l}\text { production rate/ } \\
\mu \mathrm{mol} \mathrm{g}_{\mathrm{cat}}{ }^{-1} \mathrm{~h}^{-1}\end{array}$} & \multirow[b]{2}{*}{ reference } \\
\hline & & & & & & $\mathrm{CH}_{4}$ & $\mathrm{CO}$ & $\mathrm{CH}_{3} \mathrm{OH}$ & \\
\hline \multicolumn{10}{|l|}{ methanation } \\
\hline $\mathrm{Ru}(0.8 \%) / \mathrm{TiO}_{2}$ & $\mathrm{CO}_{2} / \mathrm{H}_{2}(g, 1: 4)$ & 1 & 180 & 100 & 100 & 6980 & - & - & {$[20]$} \\
\hline $\mathrm{Ni} 0.8 \mathrm{Mg}_{0.2} \mathrm{O}(60 \%) / \mathrm{SiO}_{2}$ & $\mathrm{CO}_{2} / \mathrm{H}_{2} / \mathrm{N}_{2}(g, 1: 4: 4)$ & 1 & 300 & 87 & 99 & 20873 & - & - & {$[21]$} \\
\hline $\mathrm{Ru}(3 \%) / \mathrm{Al}_{2} \mathrm{O}_{3}$ & $\mathrm{CO}_{2} / \mathrm{H}_{2} / \mathrm{N}_{2}(g, 6: 30: 64)$ & 1 & 375 & 91 & 100 & 9924 & - & - & [19] \\
\hline $\mathrm{Ni}_{\text {coreCoshell }}(5 \%) / \mathrm{SiO}_{2}$ & $\mathrm{CO}_{2} / \mathrm{H}_{2} / \mathrm{He}(g, 6.6: 20.7: 14.7)$ & 6 & 350 & 0.92 & 3 & 5 & 137 & 14 & {$[23]$} \\
\hline \multicolumn{10}{|l|}{ methanol synthesis } \\
\hline $\mathrm{Ni}_{5} \mathrm{Ga}_{3}(17 \%) / \mathrm{SiO}_{2}$ & $\mathrm{CO}_{2} / \mathrm{H}_{2}(g, 1: 3)$ & 1 & 210 & - & - & - & - & 7803 & {$[24]$} \\
\hline $\mathrm{Ni}_{5} \mathrm{Ga}_{3}(17 \%) / \mathrm{SiO}_{2}$ & $\mathrm{CO}_{2} / \mathrm{H}_{2}(g, 1: 3)$ & 1 & 200 & $\sim 8$ & - & 16 & 5930 & 3745 & [25] \\
\hline
\end{tabular}

For the synthesis of methanol by hydrogenation of $\mathrm{CO}_{2}$, the most widely studied catalysts are based on copper or mixed copper-zinc materials $[4,8]$. The benchmark material in this category is the methanol synthesis catalysts employed in industry using $\mathrm{CO} / \mathrm{CO}_{2} / \mathrm{H}_{2}$ feeds, i.e. $\mathrm{Cu} / \mathrm{ZnO} / \mathrm{Al}_{2} \mathrm{O}_{3}$ [26]. Other related materials have been recently developed for synthesis of methanol using $\mathrm{CO}_{2}$ as the only carbon substrate, as for example $\mathrm{Cu} / \mathrm{CeO}_{x} / \mathrm{TiO}_{2}$ [27], or novel nickel-gallium [24-25] or gallium-palladium [28] nanoalloy materials, which enable high methanol productivities (see Table 2). Unlike methanation, success in methanol synthesis from $\mathrm{CO}_{2}$ and $\mathrm{H}_{2}$ is limited by thermodynamics, and fine adjustments of both catalytic systems and reaction conditions are required for moderate conversions and selectivities. Extremely high pressures might facilitate the process at moderate temperatures (theoretically, conversion $>80 \%$ at 300 bar and $\left.125^{\circ} \mathrm{C}\right)$, although under more realistic conditions $\left(\mathrm{H}_{2} / \mathrm{CO}_{2}=3: 1,50\right.$ bar and $\left.250{ }^{\circ} \mathrm{C}\right)$, selectivity to methanol might reach $68 \%$, yet at low conversions $(27 \%)$ [8]. 
The aforementioned kinetic and thermodynamic constraints leave narrow ranges of operation for feasible $\mathrm{CO}_{2}$-to-fuel technologies based on catalytic hydrogenation. Activation of the stable carbon dioxide molecule is challenging at low temperatures. An opportunity in this context relies on the use of light (preferably sunlight) to promote efficient $\mathrm{CO}_{2}$ hydrogenation under mild conditions. Efforts from several research groups have brought about alternative approaches entailing significant activity enhancements under irradiation, as outlined in this review. Relevant data including active materials and production rates are collected in Table 3.

\subsection{Production of $\mathrm{CO}$ by Light-Promoted $\mathrm{CO}_{2}$ Reaction with $\mathrm{H}_{2}$}

One of the most common products of $\mathrm{CO}_{2}$ reduction processes is $\mathrm{CO}$. In photocatalytic approaches, detection of $\mathrm{CO}$ has been occasionally ascribed to the decomposition of small amounts of adventitious organic residues present on the surface of the employed materials [29], or to the reverse Boudouard reaction $\left(\mathrm{CO}_{2}(g)+\mathrm{C}(s) \rightarrow 2 \mathrm{CO}(g)\right)$ [30]. For this reason, experimental proof of suggested $\mathrm{CO}_{2}$ reduction pathways is always desirable, especially regarding the formation of $\mathrm{CO}$ as a product at low turnovers. Spectroscopic studies of the photocatalyst surfaces are useful to identify key intermediates and to provide support to mechanisms in these processes. Tanaka and co-workers used FTIR to investigate the transformation of $\mathrm{CO}_{2}$ into $\mathrm{CO}$ in the presence of $\mathrm{H}_{2}$ on $\mathrm{ZrO}_{2}$, and observed that $\mathrm{CO}_{2}$ was first adsorbed in the form of carbonates in the dark, and later reduced into formate under irradiation [31]. The intermediate carbonate would be photo-activated by near-UV light, as suggested by phosphorescence spectroscopy. This was the basis to explain why wavelengths longer than required for $\mathrm{ZrO}_{2}$ band gap transitions $(<250 \mathrm{~nm}, 5.0 \mathrm{eV})$ were sufficient for activity, whereas other classical semiconductors (e.g. $\mathrm{TiO}_{2}, \mathrm{WO}_{3}$ or $\mathrm{ZnO}$ ) did not lead to $\mathrm{CO}$ formation [32], presumably due to little tendency for adsorption of $\mathrm{CO}_{2}$. Later, the same authors proposed the formation of transient $\left[\mathrm{CO}_{2}\right]^{--}$by irradiation of the adsorbed carbonates, and the subsequent reduction into formate by dark reaction with $\mathrm{H}_{2}$, based on EPR spectroscopy data and blank experiments [33]. In the latter step of this suggested mechanism, shown in Fig. 2, formate acts as a reducing agent enabling the transformation of gaseous $\mathrm{CO}_{2}$ into $\mathrm{CO}$ and the regeneration of carbonate species, as supported by direct reactions using deliberately adsorbed formate [31]. The validity of the reaction was further extended to other insulator solids showing capacity for carbon dioxide absorption, such as the basic $\mathrm{MgO}$, which showed activities above $1 \mu \mathrm{mol} \mathrm{g} \mathrm{cat}^{-1} \mathrm{~h}^{-1}$ (see Table 3) [34], thus confirming that activation is not caused by generation of charges by light absorption as in the case of classical semiconductor photocatalysis, but rather by excitation of adsorbed carbonates and formates [35]. The order of $\mathrm{CO}_{2}$ reduction activity on alkaline perovskites $\left(\mathrm{LiTiO}_{3}>\mathrm{NaTiO}_{3}>\mathrm{KTiO}_{3}\right)$ was also found to correlate to some extent with the measured chemisorption capacity [36]. 
Table 3 Selected data on light-promoted catalytic $\mathrm{CO}_{2}$ hydrogenation

\begin{tabular}{|c|c|c|c|c|c|c|c|c|c|c|c|c|c|}
\hline \multirow[b]{2}{*}{ catalyst } & \multirow[b]{2}{*}{ feed composition } & \multirow[b]{2}{*}{ light source } & \multirow[b]{2}{*}{$\begin{array}{l}P / \mathrm{W} \\
\left(I / \mathrm{mW} \mathrm{cm}^{-2}\right)\end{array}$} & \multirow[b]{2}{*}{$T /{ }^{\circ} \mathrm{C}$} & \multirow[b]{2}{*}{$\begin{array}{r}\chi\left(\mathrm{CO}_{2}\right) / \\
\%\end{array}$} & \multicolumn{7}{|c|}{$\begin{array}{l}\text { production rate/ } \\
\mu \mathrm{mol} \mathrm{g}_{\mathrm{cat}}^{-1} \mathrm{~h}^{-1}\end{array}$} & \multirow[t]{2}{*}{ reference } \\
\hline & & & & & & $\mathrm{CH}_{4}$ & $\mathrm{CO}$ & $\mathrm{C}_{2} \mathrm{H}_{4}$ & $\mathrm{C}_{2} \mathrm{H}_{6}$ & $\mathrm{C}_{3} \mathrm{H}_{6}$ & $\mathrm{C}_{3} \mathrm{H}_{8}$ & $\mathrm{CH}_{3} \mathrm{OH}$ & \\
\hline $\mathrm{ZrO}_{2}$ & $\mathrm{CO}_{2} / \mathrm{H}_{2}(g, 3: 1)$ & $\mathrm{Hg}$ & 500 & - & 6.0 & - & 0.550 & - & - & - & - & & [32] \\
\hline $\mathrm{ZrO}_{2}$ & $\mathrm{CO}_{2} / \mathrm{H}_{2}(g, 3: 1)$ & $\mathrm{Hg}$ & 500 & 62 & 1.9 & - & 0.722 & - & - & - & - & & [33] \\
\hline $\mathrm{ZrO}_{2}$ & $\mathrm{CO}_{2} / \mathrm{H}_{2}(g, 3: 1)$ & $\mathrm{Hg}$ & 500 & 200 & 10.1 & - & 3.4 & - & - & - & - & & [33] \\
\hline $\mathrm{MgO}$ & $\mathrm{CO}_{2} / \mathrm{H}_{2}(g, 3: 1)$ & $\mathrm{Hg}$ & 500 & - & 11.5 & - & 1.6 & - & - & - & - & & [34] \\
\hline$\beta-\mathrm{Ga}_{2} \mathrm{O}_{3}$ & $\mathrm{CO}_{2} / \mathrm{H}_{2}(g, 1: 1)$ & $\mathrm{Hg}-\mathrm{Xe}$ & 200 & - & 2.4 & - & 0.720 & - & - & - & - & & [37] \\
\hline $\mathrm{MgO}$ & $\mathrm{CO}_{2} / \mathrm{H}_{2}(g, 1: 1)$ & $\mathrm{Hg}-\mathrm{Xe}$ & 200 & - & 2.4 & - & 0.710 & - & - & - & - & & [38] \\
\hline $\mathrm{CaO}$ & $\mathrm{CO}_{2} / \mathrm{H}_{2}(g, 1: 1)$ & $\mathrm{Hg}-\mathrm{Xe}$ & 200 & - & 1.2 & - & 0.350 & - & - & - & - & & [38] \\
\hline $\mathrm{ZrO}_{2}$ & $\mathrm{CO}_{2} / \mathrm{H}_{2}(g, 1: 1)$ & $\mathrm{Hg}-\mathrm{Xe}$ & 200 & - & 0.4 & - & 0.124 & - & - & - & - & & [38] \\
\hline $\mathrm{Al}_{2} \mathrm{O}_{3}$ & $\mathrm{CO}_{2} / \mathrm{H}_{2}(g, 1: 1)$ & $\mathrm{Hg}-\mathrm{Xe}$ & 200 & - & 0.2 & - & 0.072 & - & - & - & - & & {$[38]$} \\
\hline $\mathrm{LiTaO}_{3}$ & $\mathrm{CO}_{2} / \mathrm{H}_{2}(g, 3: 1)$ & $\mathrm{Hg}-\mathrm{Xe}$ & 200 & - & 0.55 & - & 0.017 & - & - & - & - & & [36] \\
\hline $\mathrm{NaTaO}_{3}$ & $\mathrm{CO}_{2} / \mathrm{H}_{2}(g, 3: 1)$ & $\mathrm{Hg}-\mathrm{Xe}$ & 200 & - & 0.13 & - & 0.004 & - & - & - & - & & {$[36]$} \\
\hline $\mathrm{KTaO}_{3}$ & $\mathrm{CO}_{2} / \mathrm{H}_{2}(g, 3: 1)$ & $\mathrm{Hg}-\mathrm{Xe}$ & 200 & - & 0.07 & - & 0.002 & - & - & - & - & & [36] \\
\hline $\mathrm{Rh}(1 \%) / \mathrm{TiO}_{2}$ & $\mathrm{CO}_{2} / \mathrm{H}_{2}(g, 3: 1)$ & $\mathrm{Hg}$ & 500 & - & 6.2 & 0.06 & 5.1 & - & - & - & - & & [39] \\
\hline $\mathrm{Rh}(4 \%) / \mathrm{TiO}_{2}$ & $\mathrm{CO}_{2} / \mathrm{H}_{2}(g, 3: 1)$ & $\mathrm{Hg}$ & 500 & - & 0.5 & 0.4 & - & - & - & - & - & & [39] \\
\hline $\mathrm{TiO}_{2}$ & $\mathrm{CO}_{2} / \mathrm{H}_{2}(g, 1: 19)$ & UVA (365 nm) & $4 \times 15$ & 43 & - & 1.46 & 0.060 & - & 0.070 & - & - & & {$[40]$} \\
\hline $\mathrm{TiO}_{2}$ & $\mathrm{CO}_{2} / \mathrm{H}_{2}(g, 1: 19)^{[\mathrm{a}]}$ & UVA (365 nm) & $4 \times 15$ & 43 & - & 4.1 & 0.140 & - & 0.100 & - & - & & {$[40]$} \\
\hline $\mathrm{ZrO}_{2}$ & $\mathrm{CO}_{2} / \mathrm{H}_{2}(g, 1: 19)$ & UVA (254 nm) & $4 \times 15$ & 43 & - & - & 0.620 & - & - & - & - & & {$[40]$} \\
\hline $\mathrm{ZrO}_{2}$ & $\mathrm{CO}_{2} / \mathrm{H}_{2}(g, 1: 19)^{[\mathrm{a}]}$ & UVA (254 nm) & $4 \times 15$ & 43 & - & - & 0.510 & - & - & - & - & & {$[40]$} \\
\hline $\mathrm{In}_{2} \mathrm{O}_{3-x}(\mathrm{OH})_{y}$ & $\mathrm{CO}_{2} / \mathrm{H}_{2}(g, 1: 1)$ & $\mathrm{Xe}$ & $(220)$ & 150 & - & - & 0.25 & - & - & - & - & & {$[41]$} \\
\hline $\mathrm{In}_{2} \mathrm{O}_{3-x}(\mathrm{OH})_{y}$ & $\mathrm{CO}_{2} / \mathrm{H}_{2}(g, 1: 1)$ & $\mathrm{Xe}$ & $(220)$ & 150 & - & - & 15 & - & - & - & - & & {$[41]$} \\
\hline $\mathrm{Pt}(0.5 \%) / \mathrm{NaTaO}_{3}$ & $\mathrm{CO}_{2} / \mathrm{H}_{2}(g, 1: 1)^{[\mathrm{a}]}$ & $\mathrm{Xe}$ & 300 & - & - & 1.13 & 139.1 & - & - & - & - & & {$[42]$} \\
\hline $\mathrm{NaTaO}_{3}$ & $\mathrm{CO}_{2} / \mathrm{H}_{2}(g, 1: 1)^{[\mathrm{a}]}$ & $\mathrm{Xe}$ & 300 & - & - & 2.07 & 10.1 & - & - & - & - & & {$[42]$} \\
\hline $\mathrm{TiO}_{2}$ & $\mathrm{CO}_{2} / \mathrm{H}_{2} / \mathrm{He}(g, 30: 20: 50)$ & $\mathrm{Hg}$ & $(150)$ & 120 & 1.7 & 68 & 58 & 0.13 & 3.25 & 0.33 & 0.38 & & {$[43]$} \\
\hline $\mathrm{In}_{2} \mathrm{O}_{3}(3.5 \%) / \mathrm{TiO}_{2}$ & $\mathrm{CO}_{2} / \mathrm{H}_{2} / \mathrm{He}(g, 30: 20: 50)$ & $\mathrm{Hg}$ & $(150)$ & 120 & 6.42 & 31 & 2028 & 0.48 & 1.94 & 0.19 & 0.18 & & {$[43]$} \\
\hline $\mathrm{Cu}_{2} \mathrm{O}(1 \%)-\mathrm{In}_{2} \mathrm{O}_{3}(3.5 \%) / \mathrm{TiO}_{2}$ & $\mathrm{CO}_{2} / \mathrm{H}_{2} / \mathrm{He}(g, 30: 20: 50)$ & $\mathrm{Hg}$ & $(150)$ & 120 & 9.57 & 41.85 & 6540 & 0.6 & 2.82 & 1.7 & 0.88 & & {$[43]$} \\
\hline $\mathrm{NiO}(1 \%)-\mathrm{In}_{2} \mathrm{O}_{3}(3.5 \%) / \mathrm{TiO}_{2}$ & $\mathrm{CO}_{2} / \mathrm{H}_{2} / \mathrm{He}(g, 30: 20: 50)$ & $\mathrm{Hg}$ & $(150)$ & 120 & 10.2 & 34 & 12029 & 0.33 & 1.95 & 2.36 & 1.01 & - & {$[44]$} \\
\hline
\end{tabular}




\begin{tabular}{|c|c|c|c|c|c|c|c|c|c|c|c|c|c|}
\hline$\left[\mathrm{Zn}_{3} \mathrm{Al}(\mathrm{OH})_{8}\right]_{2}\left[\mathrm{CO}_{3}\right]$ & $\mathrm{CO}_{2} / \mathrm{H}_{2}(g, \sim 1: 9.4)$ & $\mathrm{Xe}$ & $500^{[\mathrm{b}]}$ & - & 0.16 & - & 0.620 & - & - & - & - & 0.039 & {$[45]$} \\
\hline$\left[\mathrm{Zn}_{1.5} \mathrm{Cu}_{1.5} \mathrm{Al}(\mathrm{OH})_{8}\right]_{2}\left[\mathrm{CO}_{3}\right]$ & $\mathrm{CO}_{2} / \mathrm{H}_{2}(g, \sim 1: 9.4)$ & $\mathrm{Xe}$ & $500^{[\mathrm{c}]}$ & - & 0.16 & - & 0.580 & - & - & - & - & 0.200 & {$[45]$} \\
\hline$\left[\mathrm{Zn}_{1.5} \mathrm{Cu}_{1.5} \mathrm{Al}(\mathrm{OH})_{8}\right]_{2}\left[\mathrm{CO}_{3}\right]$ & $\mathrm{CO}_{2} / \mathrm{H}_{2}(g, \sim 1: 9.4)$ & $\mathrm{Xe}$ & $500^{[b]}$ & - & 0.11 & - & 0.370 & - & - & - & - & 0.130 & {$[45]$} \\
\hline$\left[\mathrm{Zn}_{3} \mathrm{Ga}(\mathrm{OH})_{8}\right]_{2}\left[\mathrm{CO}_{3}\right]$ & $\mathrm{CO}_{2} / \mathrm{H}_{2}(g, \sim 1: 9.4)$ & $\mathrm{Xe}$ & $500^{[\mathrm{b}]}$ & - & 0.02 & - & 0.080 & - & - & - & - & 0.051 & {$[45]$} \\
\hline$\left[\mathrm{Zn}_{1.5} \mathrm{Cu}_{1.5} \mathrm{Ga}(\mathrm{OH})_{8}\right]_{2}\left[\mathrm{CO}_{3}\right]$ & $\mathrm{CO}_{2} / \mathrm{H}_{2}(g, \sim 1: 9.4)$ & $\mathrm{Xe}$ & $500^{[b]}$ & - & 0.03 & - & 0.079 & - & - & - & - & 0.170 & {$[45]$} \\
\hline $\mathrm{Cu}-\mathrm{ZnO}(\mathrm{Cu}: \mathrm{Zn}=54: 46)$ & $\mathrm{CO}_{2} / \mathrm{H}_{2}(g, \sim 1: 9.4)$ & $\mathrm{Xe}$ & $500^{[\mathrm{b}]}$ & - & 0.006 & - & 0.030 & - & - & - & - & - & {$[45]$} \\
\hline$\alpha-\mathrm{Ga}_{2} \mathrm{O}_{3}$ & $\mathrm{CO}_{2} / \mathrm{H}_{2}(g, \sim 1: 9.4)$ & $\mathrm{Xe}$ & $500^{[\mathrm{b}]}$ & - & 0.01 & - & 0.047 & - & - & - & - & - & {$[45]$} \\
\hline$\left[\mathrm{Zn}_{3} \mathrm{Ga}(\mathrm{OH})_{8}\right]_{2}\left[\mathrm{Cu}(\mathrm{OH})_{4}\right]$ & $\mathrm{CO}_{2} / \mathrm{H}_{2}(g, \sim 1: 9.4)$ & $\mathrm{Xe}$ & $500^{[\mathrm{d}]}$ & - & 0.04 & - & 0.130 & - & - & - & - & 0.300 & {$[46]$} \\
\hline$\left[\mathrm{Zn}_{1.5} \mathrm{Cu}_{1.5} \mathrm{Ga}(\mathrm{OH})_{8}\right]_{2}\left[\mathrm{Cu}(\mathrm{OH})_{4}\right]$ & $\mathrm{CO}_{2} / \mathrm{H}_{2}(g, \sim 1: 9.4)$ & $\mathrm{Xe}$ & $500^{[\mathrm{d}]}$ & - & 0.05 & - & 0.070 & - & - & - & - & 0.490 & [46] \\
\hline Ti-SBA-15 & $\mathrm{CO}_{2} / \mathrm{H}_{2}(g, 1: 2)$ & $\mathrm{Hg}$ & 120 & 40 & 0.02 & 0.012 & - & 0.008 & 0.005 & - & - & - & [47] \\
\hline $\mathrm{Ru} / \mathrm{RuO}_{2}(3.8 \%) / \mathrm{TiO}_{2}{ }^{[\mathrm{ee}]}$ & $\mathrm{CO}_{2} / \mathrm{H}_{2} / \mathrm{Ar}(g, 5: 60: 35)$ & solar simulator & $(80)$ & 46 & 98 & 51.8 & - & - & - & - & - & - & {$[48]$} \\
\hline $\mathrm{Ru} / \mathrm{SiO}_{2}$ (glass) & $\mathrm{CO}_{2} / \mathrm{H}_{2}(g, 1: 4)$ & $\mathrm{Xe}$ & $(320)$ & 150 & - & 75.2 & - & - & - & - & - & - & [49] \\
\hline $\mathrm{Ru} / \mathrm{Si}$ & $\mathrm{CO}_{2} / \mathrm{H}_{2}(g, 1: 4)$ & $\mathrm{Xe}$ & $(320)$ & 150 & - & 140 & - & - & - & - & - & - & [49] \\
\hline $\mathrm{Ru} / \mathrm{Si}$ (nanowires) & $\mathrm{CO}_{2} / \mathrm{H}_{2}(g, 1: 4)$ & $\mathrm{Xe}$ & $(320)$ & 150 & - & 990 & - & - & - & - & - & - & [49] \\
\hline $\mathrm{Ru}(2.4 \%) / \mathrm{Al}_{2} \mathrm{O}_{3}$ & $\mathrm{CO}_{2} / \mathrm{H}_{2}(g, 1: 4.1)$ & $\mathrm{Xe}$ & 300 & 370 & 95.8 & 300000 & - & - & - & - & - & - & {$[50]$} \\
\hline $\mathrm{Rh}(2.6 \%) / \mathrm{Al}_{2} \mathrm{O}_{3}$ & $\mathrm{CO}_{2} / \mathrm{H}_{2}(g, 1: 4.1)$ & $\mathrm{Xe}$ & 300 & 370 & 96.3 & 156000 & - & - & - & - & - & - & [50] \\
\hline $\mathrm{Ni}(2.1 \%) / \mathrm{Al}_{2} \mathrm{O}_{3}$ & $\mathrm{CO}_{2} / \mathrm{H}_{2}(g, 1: 4.1)$ & $\mathrm{Xe}$ & 300 & 380 & 93.3 & 48000 & 3000 & - & - & - & - & - & {$[50]$} \\
\hline $\mathrm{Co}(2.5 \%) / \mathrm{Al}_{2} \mathrm{O}_{3}$ & $\mathrm{CO}_{2} / \mathrm{H}_{2}(g, 1: 4.1)$ & $\mathrm{Xe}$ & 300 & 350 & 92.6 & 21000 & 6000 & - & - & - & - & - & {$[50]$} \\
\hline $\mathrm{Pd}(2.0 \%) / \mathrm{Al}_{2} \mathrm{O}_{3}$ & $\mathrm{CO}_{2} / \mathrm{H}_{2}(g, 1: 4.1)$ & $\mathrm{Xe}$ & 300 & 370 & 93.4 & 8000 & 2000 & - & - & - & - & - & {$[50]$} \\
\hline $\mathrm{Pt}(2.4 \%) / \mathrm{Al}_{2} \mathrm{O}_{3}$ & $\mathrm{CO}_{2} / \mathrm{H}_{2}(g, 1: 4.1)$ & $\mathrm{Xe}$ & 300 & 350 & 60.4 & 600 & 9600 & - & - & - & - & - & {$[50]$} \\
\hline $\mathrm{Ir}(2.8 \%) / \mathrm{Al}_{2} \mathrm{O}_{3}$ & $\mathrm{CO}_{2} / \mathrm{H}_{2}(g, 1: 4.1)$ & $\mathrm{Xe}$ & 300 & 320 & 14.9 & 720 & 640 & - & - & - & - & - & {$[50]$} \\
\hline $\mathrm{Fe}(2.4 \%) / \mathrm{Al}_{2} \mathrm{O}_{3}$ & $\mathrm{CO}_{2} / \mathrm{H}_{2}(g, 1: 4.1)$ & $\mathrm{Xe}$ & 300 & 320 & 7.3 & 30 & 420 & - & - & - & - & - & {$[50]$} \\
\hline $\mathrm{Al}_{2} \mathrm{O}_{3}$ & $\mathrm{CO}_{2} / \mathrm{H}_{2}(g, 1: 4.1)$ & $\mathrm{Xe}$ & 300 & 150 & - & - & 1.92 & - & - & - & - & - & {$[50]$} \\
\hline $\mathrm{Ni} / \mathrm{SiO}_{2} \cdot \mathrm{Al}_{2} \mathrm{O}_{3}$ & $\mathrm{CO}_{2} / \mathrm{H}_{2}(g, 1: 4.6)$ & solar simulator & - & 150 & 94.9 & 54608 & 1573 & - & - & - & - & - & [51] \\
\hline $\mathrm{NiO}$ & $\mathrm{CO}_{2} / \mathrm{H}_{2}(g, 1: 4.6)$ & solar simulator & - & 150 & 89.8 & 13290 & - & - & - & - & - & - & {$[51]$} \\
\hline $\mathrm{Fe}_{2} \mathrm{O}_{3}$ & $\mathrm{CO}_{2} / \mathrm{H}_{2}(g, 1: 4.6)$ & solar simulator & - & 150 & 51 & 325 & 7171 & - & 53 & - & - & - & {$[51]$} \\
\hline $\mathrm{CoO}$ & $\mathrm{CO}_{2} / \mathrm{H}_{2}(g, 1: 4.6)$ & solar simulator & - & 150 & 27.4 & 1569 & 2453 & - & 32 & - & - & - & [51] \\
\hline
\end{tabular}

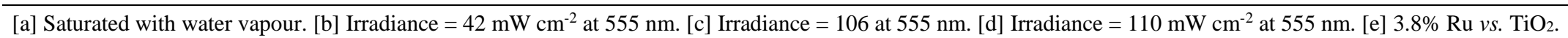



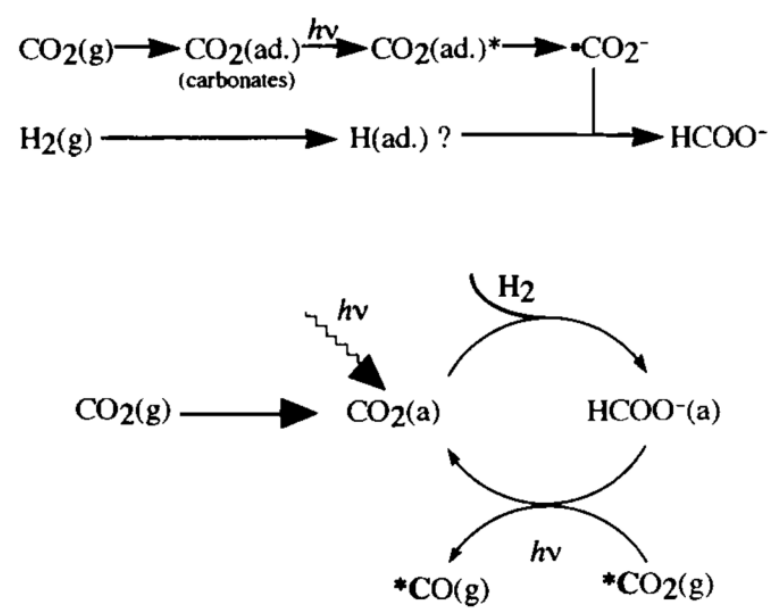

Fig. 2 Suggested mechanism for the light-induced reduction of $\mathrm{CO}_{2}$ to $\mathrm{CO}$ in the presence of $\mathrm{H}_{2}$ on solids such as $\mathrm{ZrO}_{2}$ or $\mathrm{MgO}$. The proposed activation steps (top) include adsorption as carbonates, absorption of light by these species, one-electron reduction on the solid, and further reduction by $\mathrm{H}_{2}$ into formate; both activation and $\mathrm{CO}$ release by reaction between formate and gaseous $\mathrm{CO}_{2}$ are also schematically summarised (bottom). Reproduced from reference [33] (@) The Owner Societies, 2000).

A screening on several oxides performed by the same research team revealed that, in addition to other basic oxides such as $\mathrm{CaO}$, the activity was surprisingly high using $\mathrm{Ga}_{2} \mathrm{O}_{3}$ [37-38]. In contrast to the mechanism on $\mathrm{ZrO}_{2}$ or $\mathrm{MgO}$, heterolytic dissociation of $\mathrm{H}_{2}$ on $\mathrm{Ga}_{2} \mathrm{O}_{3}$ (observed by FTIR spectroscopy), and direct influence of its partial pressure on reaction rates following a Langmuir-Hinshelwood model, pointed to a completely different activation route [37].

Regarding classical photocatalysts, Tanaka and co-workers observed that $\mathrm{Rh} / \mathrm{TiO}_{2}$ produced $\mathrm{CO}$ and traces of $\mathrm{CH}_{4}$, as opposed to the otherwise inactive bare titania [39]. The presence of deposited rhodium was found to enable reduction, even in the dark to a little extent, yet absorption of light by the semiconductor was required, as proven by the lack of activity of analogous $\mathrm{Rh} / \mathrm{SiO}_{2}$. Other researchers have confirmed the production of $\mathrm{CO}$ by light-promoted (254 nm) reduction of $\mathrm{CO}_{2}$ on $\mathrm{ZrO}_{2}$ in the presence of $\mathrm{H}_{2}$ at $43{ }^{\circ} \mathrm{C}$ [40]. In the same study, it was proven that $\mathrm{TiO}_{2}$ becomes active under band gap irradiation $(365 \mathrm{~nm})$ especially when the gas feed is saturated with water vapour (see Table 3), and that the selectivity is switched to the preferential production of methane [40]. Similarly, combined beneficial effects of $\mathrm{H}_{2} / \mathrm{H}_{2} \mathrm{O}$ presence have been recently claimed for photocatalysts based on $\mathrm{NaTiO}_{3}$, whereby peroxides generated by water oxidation would be in turn annihilated by hydrogen [42]. Metal deposition of such materials was shown to enhance activity, with platinum and ruthenium favouring the formation of either $\mathrm{CO}$ or $\mathrm{CH}_{4}$, respectively.

The production of $\mathrm{CO}$ by hydrogenation of $\mathrm{CO}_{2}$ under $\mathrm{UV}$-vis light on titania-based materials may be greatly enhanced by increasing the temperature, as demonstrated by Tahir and Amin [43-44]. These researchers found an optimum operation temperature of $120^{\circ} \mathrm{C}$. In addition, they claimed the process to be initiated by light, given that no products were observed during 
experiments in the dark in the $120-150{ }^{\circ} \mathrm{C}$ range. Regarding photocatalysts, bare $\mathrm{TiO}_{2}$ was related to decreased $\mathrm{CO}$ selectivities and preferential formation of $\mathrm{CH}_{4}$ and other alkanes, whereas the presence of indium or indium/copper co-catalysts boosted $\mathrm{CO}$ productivities (see Table 3) [43]. Similar effects were later reported for analogous indium/nickel materials [44]. Single-component indium oxide-hydroxides exhibiting activity for the reduction of $\mathrm{CO}_{2}$ into $\mathrm{CO}$ by $\mathrm{H}_{2}$ under simulated solar light have been developed by Ozin and co-workers [41]. The optimum temperature was $150{ }^{\circ} \mathrm{C}$. Significant endeavours were undertaken to elucidate the real influence of light in the promotion of the reaction, a fact which was proven by measuring the enhanced $\mathrm{CO}$ production rates at a fixed temperature under increasing irradiances, and the lower activities in the dark.

\subsection{Production of Methanol by Light-Promoted Hydrogenation of $\mathrm{CO}_{2}$}

As discussed above, methanol is an easily transportable liquid fuel and chemical feedstock, and as such, its production by reduction of $\mathrm{CO}_{2}$ is preferred for many processes over other contenders. Achieving high methanol selectivities is challenging due to thermodynamic and mechanistic limitations. In the context of photocatalytic $\mathrm{CO}_{2}$ transformations, many researchers have focused on designing materials and irradiation systems for methanol production [13-14].

The family of layered double hydroxides comprises hexagonal structures formed by positively charged layers held together by electrostatic interactions with intercalating anions (or solvated anions) [13]. Izumi and co-workers have studied zinc- or zinc/copper-based layered double hydroxides paired with carbonate anions $\left(\left[\mathrm{Zn}_{3-x} \mathrm{Cu}_{x} \mathrm{M}^{\mathrm{III}}(\mathrm{OH})_{8}\right]_{2}\left[\mathrm{CO}_{3}\right] \cdot m \mathrm{H}_{2} \mathrm{O}\right.$, where $\mathrm{M}^{\mathrm{III}}=\mathrm{Al}$ or $\mathrm{Ga}$, and $x=0$ or 1.5 ) as photocatalysts for the hydrogenation of $\mathrm{CO}_{2}$ to methanol under $\mathrm{UV}$-vis light, with formation of $\mathrm{CO}$ as a co-product $[45-46,52]$. Based on their screening, the most active material was $\left[\mathrm{Zn}_{3} \mathrm{Al}(\mathrm{OH})_{8}\right]_{2}\left[\mathrm{CO}_{3}\right] \cdot m \mathrm{H}_{2} \mathrm{O}(0.16 \%$ conversion after $5 \mathrm{~h})$, albeit the main product was CO [45]. Introduction of copper into the structures rendered the solids slightly less active yet more selective (to methanol), and further improvements were achieved by using gallium counterparts. The reversible reducibility of copper and the modified (narrower) band gaps induced by its inclusion were claimed as causes to the improved performances. The authors proposed the formation of hydrogencarbonate at active copper (or copper-gallium) sites, and their subsequent hydrogenation as the key factors determining selectivity, based on XANES and FTIR measurements [45, 52]. Despite the rather low formation rates $\left(<1 \mu \mathrm{mol} \mathrm{g}_{\mathrm{cat}}{ }^{-1} \mathrm{~h}^{-1}\right.$, see Table 3), the advances made by these researchers towards the challenging production of methanol by photocatalytic reduction of $\mathrm{CO}_{2}$ are noteworthy. Conversions using mixed copperzinc oxides (reminiscent of commercial methanol synthesis catalysts) or gallium(III) oxide were significantly lower than those found for layered double hydroxides [45]. Furthermore, only CO was formed on these reference catalysts, a fact which is consistent with previous data for $\mathrm{Ga}_{2} \mathrm{O}_{3}$ [37]. 
Additional improvements in methanol productivity $\left(0.49 \mu \mathrm{mol} \mathrm{g}_{\mathrm{cat}}{ }^{-1} \mathrm{~h}^{-1}\right.$, see Table 3$)$ were achieved by replacing inter-layer carbonate with tetrahydroxycuprate anions $\left(\left[\mathrm{Cu}(\mathrm{OH})_{4}\right]^{2-}\right)$ in the zinc-gallium or zinc-copper-gallium materials [46]. The selectivity to methanol reached a maximum at $88 \%$ for $\left[\mathrm{Zn}_{1.5} \mathrm{Cu}_{1.5} \mathrm{Al}(\mathrm{OH})_{8}\right]_{2}\left[\mathrm{Cu}(\mathrm{OH})_{4}\right] \cdot m \mathrm{H}_{2} \mathrm{O}$, presumably due to the more active copper centres provided by the anions. In this study, the authors also proved that pretreatment of the photocatalyst under vacuum led to activity improvements due to partial removal of solvating water molecules, and consequently facilitated diffusion of carbon dioxide.

In spite of the relatively low conversions and yields, the work performed by Izumi and coworkers proves the possibility of transforming $\mathrm{CO}_{2}$ to methanol by the action of light on appropriate photocatalytic materials. Further investigations on this topic are expected to contribute to strategies for recycling carbon dioxide to fuels and chemicals.

\subsection{Light-Promoted Methanation of $\mathrm{CO}_{2}$}

The production of variable amounts of $\mathrm{CH}_{4}$ by methanation of carbon dioxide under irradiation has been reported by several researchers. For example, $\mathrm{TiO}_{2}$ has been shown to enhance selectivities to methane over carbon dioxidemonoxide, with minor amounts of ethane also formed (see Table 3) [40]. Methane and other $C_{2}$ and $C_{3}$ hydrocarbons have been observed on titania-based [43-44] or $\mathrm{NaTiO}_{3}$ [42] photocatalysts. On the other hand, Mul and co-workers described the production of methane, ethane and ethylene on titanium-containing mesoporous silica materials (Ti-SBA-15) under UV-vis light [47].

Enhancements in the reduction of $\mathrm{CO}_{2}$ by $\mathrm{H}_{2}$ to $\mathrm{CH}_{4}$ (i.e. methanation of carbon dioxide, or Sabatier reaction [53]) were first reported by Grätzel and co-workers by using $\mathrm{Ru} / \mathrm{RuO}_{x} / \mathrm{TiO}_{2}$ materials irradiated by means of a solar simulator [48]. In that pioneering study, conversion of $\mathrm{CO}_{2}$ and formation of $\mathrm{CH}_{4}$ were observed under irradiation $\left(80 \mathrm{~mW} \mathrm{~cm}^{-2}\right.$, see Table_3); a significant temperature increase from ambient to $46{ }^{\circ} \mathrm{C}$ was also noted. Since dark conditions also resulted in measurable activity, the authors designed experiments to elucidate whether or not the reaction was activated by light to some extent. First, they recorded a lower yield at the same temperature in the absence of light, as show in Fig. 3. Then, they proved that the initial methanation rates only increased by using near-UV light, based on data from irradiations using a water jacket (to remove IR frequencies) and several cut-off filters $(<435 \mathrm{~nm})$. These evidences led to conclude that absorption of light by the semiconductor $\left(\mathrm{TiO}_{2}\right)$ played a role in promoting the reaction by the photo-generation of charges, thus adding up to the effects of thermal activation [48]. 


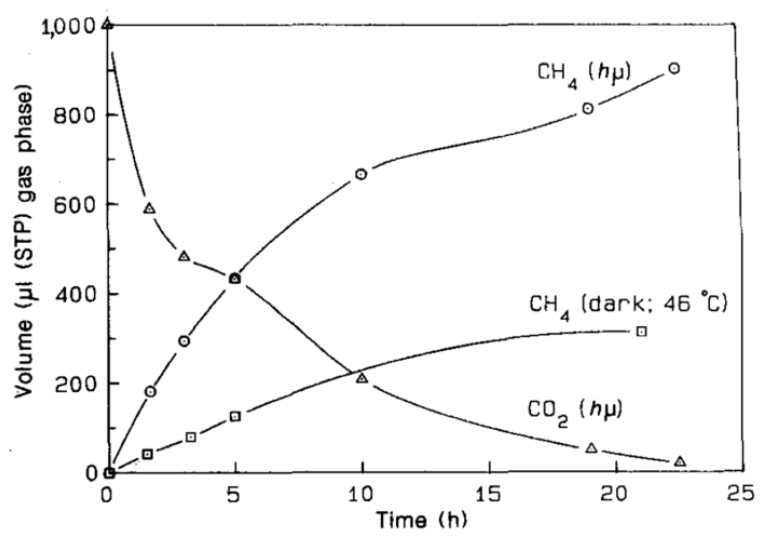

Fig. 3 Time profiles of $\mathrm{CO}_{2}$ methanation reactions on $\mathrm{Ru} / \mathrm{RuO}_{x} / \mathrm{TiO}_{2}$ either under irradiation $\left(\mathrm{CO}_{2} / \mathrm{H}_{2} / \mathrm{Ar}=\right.$ 0.05:0.60:0.35 atm, solar simulator, $80 \mathrm{~mW} \mathrm{~cm}^{-12}, 46^{\circ} \mathrm{C}$ ) or in the dark under analogous conditions. Reproduced from reference [48] (@ MacMillan Publishers Limited, 1987).

A later thorough investigation revisited the results of Grätzel and co-workers and concluded that only thermal effects, to some extent caused by irradiation, were responsible for the methanation

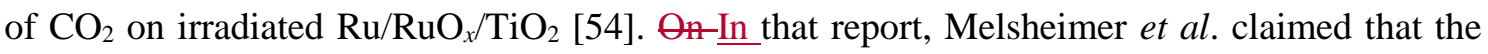
enhancement in $\mathrm{CH}_{4}$ production was effectively suppressed by passing the beam through a $25 \mathrm{~mm}$ (optical length) water filter, even when varying irradiance from 2 to $350 \mathrm{~mW} \mathrm{~cm}^{-12}$. Also in contrast to Grätzel's data, the use of glass filtering off wavelengths below ca. $320 \mathrm{~nm}$ had no effect, a fact which suggested that the reaction was not photocatalytic.

In line with the use of ruthenium catalysts for light-promoted methanation of $\mathrm{CO}_{2}$, Ozin and coworkers have recently reported the preparation of novel $\mathrm{Ru} / \mathrm{Si}$ nanomaterials whereby ruthenium was deposited in the form of small nanoparticles by sputtering on silicon nanowires [49]. These materials outperformed counterparts based on silicon wafer supports (990 and $140 \mu \mathrm{mol} \mathrm{gcat}^{-1} \mathrm{~h}^{-1}$, respectively, see Table 3), presumably as a consequence of a superior light absorbance. Activities were also noticeable in the dark, as mentioned above for other ruthenium catalysts. Therefore, the authors performed tests while carefully monitoring temperatures to discern between thermal and radiative activation. They observed that reaction rates were directly dependent on photon flux under different wavelength ranges at a constant temperature $\left(93^{\circ} \mathrm{C}\right)$, and moreover, that enhancement increases with heating were more marked under irradiation, yet activation energies (and thus, mechanistic routes) appeared to be identical. With these evidences in hand, the process was claimed to be genuinely promoted, at least in part, by photocatalysis [49].

The possibility of achieving high efficiencies - superior to those of typical catalytic processesby virtue of sunlight-like irradiation for the production of synthetic natural gas by hydrogenation of $\mathrm{CO}_{2}$ has been recently explored using a range of Group VIII metallic nanomaterials [50]. Activities depended strongly on the metal, specifically in the $\mathrm{Ru}>\mathrm{Rh}>\mathrm{Ni}$ $>\mathrm{Co}>\mathrm{Pd}>\mathrm{Pt}>>\mathrm{Ir}>\mathrm{Fe}$ order (see Table 3 ), for alumina-supported catalysts prepared by 
impregnation. Reaction rates were exceptionally elevated, especially for ruthenium. Selectivities to methane were noticeably high, except for Pt, Ir and Fe. Temperature monitoring revealed that substantial heating (up to $350-400{ }^{\circ} \mathrm{C}$ ) occurred upon irradiation, a phenomenon which was ascribed to the photothermal effect of metal nanoparticles, that is, the transformation of radiative energy into heat. Experiments in the dark confirmed this, and the authors thus claimed that no true photocatalysis (rather, only thermal activation) was taking place. However, the semiconductor-based $\mathrm{Ni} / \mathrm{TiO}_{2}$ performed better than $\mathrm{Ni} / \mathrm{Al}_{2} \mathrm{O}_{3}$ under irradiation [50].

In line with the aforementioned report, Fu and co-workers demonstrated that the hydrogenation of $\mathrm{CO}$ on $\mathrm{M} / \mathrm{TiO}_{2}(\mathrm{M}=\mathrm{Ru}$ [55] or $\mathrm{Ni}[56])$ catalysts at moderate temperatures can be further promoted by UV irradiation. This was confirmed by the lack of effect in the case an analogous catalyst based on alumina, which being an insulator, cannot contribute via a photocatalytic pathway. Spectroscopic (XPS and FTIR) measurements revealed that CO adsorbed on the metallic surface underwent activation on the electron-enriched irradiated samples.

Corma and co-workers have investigated on the transformation of $\mathrm{CO}$ or $\mathrm{CO}_{2}$ by different semiconductor oxides under simulated solar irradiation. On an initial stage, it was demonstrated that the water-gas shift reaction could be photocatalysed on titania-based materials, among which $\mathrm{Au} / \mathrm{TiO}_{2}$ enabled high conversions [57]. Subsequently, a screening of $n$ - and p-type semiconductors for the $\mathrm{CO}$ hydrogenation reaction under visible light was performed [58]. Data derived from that study revealed that, whereas n-type semiconductors $\left(\right.$ e.g. $\left.\mathrm{TiO}_{2}\right)$ favoured the water-gas shift reaction, p-type counterparts (e.g. NiO) led to the formation of methane. Time monitoring suggested that $\mathrm{CO}$ and $\mathrm{H}_{2} \mathrm{O}$ reacted to yield $\mathrm{CO}_{2}$ and $\mathrm{H}_{2}$ initially, yet at longer reaction times, hydrogen was consumed giving rise to $\mathrm{CH}_{4}$ formation. Surprisingly, the reaction between $\mathrm{CO}$ and $\mathrm{H}_{2}$ resulted in the evolution of $\mathrm{CH}_{4}$ and $\mathrm{O}_{2}$. These evidences, in conjunction with photo-action spectra, suggested that $\mathrm{CO}$ was readily reduced on p-type semiconductors. Later, it was also demonstrated that $\mathrm{CO}_{2}$ methanation proceeded at high rates on nanoparticulated nickel materials under simulated solar light [51]. Other p-type semiconductors, such as $\mathrm{CoO}, \mathrm{Fe}_{2} \mathrm{O}_{3}$ or $\mathrm{Fe}_{3} \mathrm{O}_{4}$ mainly produced $\mathrm{CO}$, although $\mathrm{CH}_{4}$ and $\mathrm{C}_{2} \mathrm{H}_{6}$ were also formed in smaller amounts. Striking activities (95\% conversion, > 97\% selectivity, after only $15 \mathrm{~min}$ of irradiation) and reusability (up to six cycles performed by evacuating the system and then adding fresh feed gases) for the methanation reaction was-were reported for $\mathrm{Ni} / \mathrm{SiO}_{2} \cdot \mathrm{Al}_{2} \mathrm{O}_{3}$ catalysts. The activity was only reduced to a quarter of the original by filtering off the UV portion of the incident light. Although temperature increases (up to around $150{ }^{\circ} \mathrm{C}$ ) were noted, negligible activity was observed in the dark, a fact which - in conjunction to the detection of photo-generated charges by laser flash photolysis experiments and to photo-action spectrasuggested the existence of genuine light-promotion for the process. 
It is apparent from the various examples in the literature that irradiation under appropriate conditions promotes the hydrogenation of $\mathrm{CO}_{2}$ on supported metal (especially ruthenium, rhodium and-ornickel) catalysts. Both photothermal and photocatalytic processes might be at play. Elucidating the extent of each effect remains challenging, due to the difficulty in unambiguously probing the real contributions of heat and light. Regardless of the exact activation routes, the beneficial effects of solar light on the outcome of $\mathrm{CO}_{2}$-to-fuel processes have been largely proven. Provided hydrogen is produced from renewable resources and energy, as depicted in Fig. 4, this strategy would become sustainable $[3,50]$.

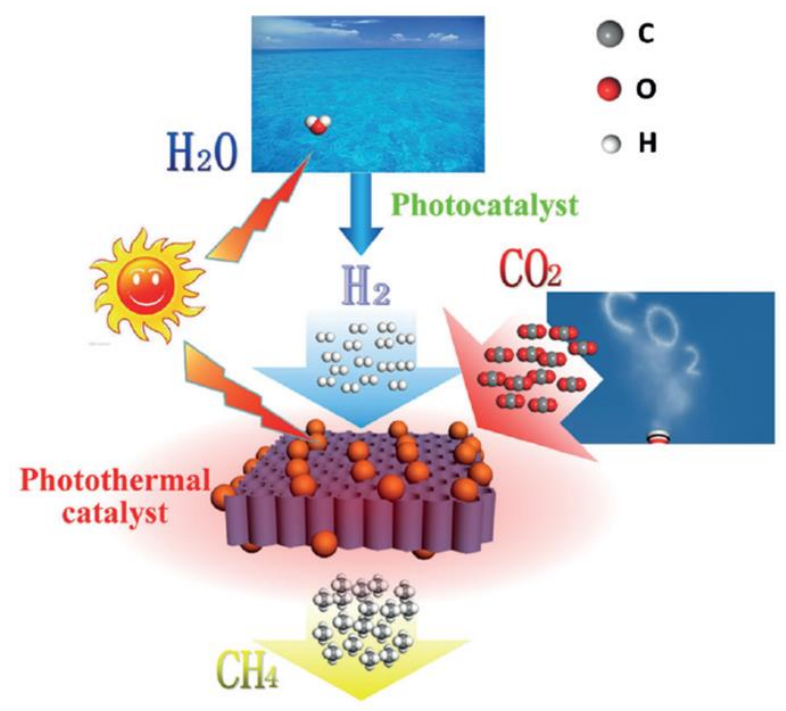

Fig. 4 A schematic view of a sunlight-driven $\mathrm{CO}_{2}$-to-fuel strategy, including the use of solar energy for both the production of $\mathrm{H}_{2}$ and the methanation of $\mathrm{CO}_{2}$. Reproduced from reference [50] (C) Wiley, 1987).

\section{$4 \quad$ Conclusions and Prospects}

Solar fuels, that is, substances which enable the storage of sunlight energy in the form of transportable chemical energy, are at the centre of key technologies for sustainable development away from the exploitation of finite, fossil resources. Carbon dioxide can be used as the feedstock for solar fuels via appropriate reduction processes, among which, hydrogenation is capable to provide a range of substances compatible with current supply schemes. Provided that the hydrogen required is obtained from renewable energy and raw materials, the transformation into fuels such as methane or methanol is an ideal strategy to reduce net emissions of carbon dioxide. Finally, if solar energy is used to promote these processes, requirement of fossil carbon-based raw materials is virtually reduced to a minimum.

State-of-the-art methods for hydrogenation of carbon dioxide using sunlight as the main energy input have proven to be highly efficient, even outperforming the known, commercially relevant, heat-activated competitor processes. The production of synthetic natural gas (or solar methane) 
from carbon dioxide and hydrogen on appropriate nanomaterials takes place at high conversions and selectivities (both above 95\%) and at formidably fast rates (> $10^{4} \mu \mathrm{mol} \mathrm{g}_{\mathrm{cat}}{ }^{-1} \mathrm{~h}^{-1}$ ). Materials based on ruthenium, rhodium or nickel nanoparticles have been claimed as the best performers in light-promoted methanation. On the other hand, layered double hydroxides containing zinc, copper and gallium enable high methanol selectivities under UV-visible light, albeit at low conversions. Other semiconductor oxides perform moderately as photocatalysts for the reverse water-gas shift to produce carbon monoxide. The mechanisms of activation for the most active processes, especially methanation on nano-sized metallic materials, are currently under debate. Local heating of the catalyst surface by action of solar-like irradiation has been claimed as the main factor leading to fast rates and efficient conversion, whereas experiments using semiconductors as components of the active materials suggest that genuine charge separation across their band gaps - i.e. via photocatalysis - might be also at play. Further investigations in this field of research are desirable in order to clarify the relevance of actual activation routes and to design revolutionary nanomaterials for appealing solar fuel technologies.

\section{References}

1. Centi G, Perathoner S (2009) Opportunities and prospects in the chemical recycling of carbon dioxide to fuels. Catal Today 148:191-205

2. Aresta M, Dibenedetto A, Angelini A (2014) Catalysis for the Valorization of Exhaust Carbon: from $\mathrm{CO}_{2}$ to Chemicals, Materials, and Fuels. Technological Use of $\mathrm{CO}_{2}$. Chem Rev 114:1709-1742

3. Centi G, Quadrelli EA, Perathoner S (2013) Catalysis for $\mathrm{CO}_{2}$ conversion: a key technology for rapid introduction of renewable energy in the value chain of chemical industries. Energy Environ Sci 6:1711-1731

4. Wang W, Wang S, Ma X, Gong J (2011) Recent advances in catalytic hydrogenation of carbon dioxide. Chem Soc Rev 40:3703-3727

5. Gao J, Liu Q, Gu F, Liu B, Zhong Z, Su F (2015) Recent advances in methanation catalysts for the production of synthetic natural gas. RSC Adv 5:22759-22776

6. Armaroli N, Balzani V (2011) The Hydrogen Issue. ChemSusChem 4:21-36

7. Gao J, Wang Y, Ping Y, Hu D, Xu G, Gu F, Su F (2012) A thermodynamic analysis of methanation reactions of carbon oxides for the production of synthetic natural gas. RSC Adv 2:2358-2368

8. Jadhav SG, Vaidya PD, Bhanage BM, Joshi JB (2014) Catalytic carbon dioxide hydrogenation to methanol: A review of recent studies. Chem Eng Res Des 92:2557-2567

9. de_Richter RK, Ming T, Caillol S (2013) Fighting global warming by photocatalytic reduction of $\mathrm{CO}_{2}$ using giant photocatalytic reactors. Renew Sust Energ Rev 19:82-106

10. Schach M-O, Schneider R, Schramm H, Repke J-U (2010) Techno-Economic Analysis of Postcombustion Processes for the Capture of Carbon Dioxide from Power Plant Flue Gas. Ind. Eng. Chem. Res. 49:2363-2370

11. Centi G, Perathoner S (2010) Towards Solar Fuels from Water and $\mathrm{CO}_{2}$. ChemSusChem 3:195-208

12. Corma A, Garcia $\mathrm{H}$ (2013) Photocatalytic reduction of $\mathrm{CO}_{2}$ for fuel production: Possibilities and challenges. J Catal 308:168-175

13. Izumi Y (2013) Recent advances in the photocatalytic conversion of carbon dioxide to fuels with water and/or hydrogen using solar energy and beyond. Coord Chem Rev 257:171-186 
14. Dhakshinamoorthy A, Navalon S, Corma A, Garcia H (2012) Photocatalytic $\mathrm{CO}_{2}$ reduction by $\mathrm{TiO}_{2}$ and related titanium containing solids. Energy Environ Sci 5:9217-9233

15. Indrakanti VP, Kubicki JD, Schobert $\mathrm{HH}$ (2009) Photoinduced activation of $\mathrm{CO}_{2}$ on Tibased heterogeneous catalysts: Current state, chemical physics-based insights and outlook. Energy Environ Sci 2:745-758

16. Ozin GA (2015) You can't have an energy revolution without transforming advances in materials, chemistry and catalysis into policy change and action. Energy Environ Sci 8:16821684

17. Ozin GA (2015) Throwing New Light on the Reduction of $\mathrm{CO}_{2}$. Adv Mater 27:19571963

18. Garbarino G, Riani P, Magistri L, Busca G (2014) A study of the methanation of carbon dioxide on $\mathrm{Ni} / \mathrm{Al}_{2} \mathrm{O}_{3}$ catalysts at atmospheric pressure. Int $\mathrm{J}$ Hydrogen Energy 39:11557-11565

19. Garbarino G, Bellotti D, Riani P, Magistri L, Busca G (2015) Methanation of carbon dioxide on $\mathrm{Ru} / \mathrm{Al}_{2} \mathrm{O}_{3}$ and $\mathrm{Ni} / \mathrm{Al}_{2} \mathrm{O}_{3}$ catalysts at atmospheric pressure: Catalysts activation, behaviour and stability. Int J Hydrogen Energy 40:9171-9182

20. Abe T, Tanizawa M, Watanabe K, Taguchi A (2009) $\mathrm{CO}_{2}$ methanation property of $\mathrm{Ru}$ nanoparticle-loaded $\mathrm{TiO}_{2}$ prepared by a polygonal barrel-sputtering method. Energy Environ Sci 2:315-321

21. Li Y, Lu G, Ma J (2014) Highly active and stable nano NiO-MgO catalyst encapsulated by silica with a core-shell structure for $\mathrm{CO}_{2}$ methanation. RSC Adv 4:17420-17428

22. Iablokov V, Beaumont SK, Alayoglu S, Pushkarev VV, Specht C, Gao J, Alivisatos AP, Kruse N, Somorjai GA (2012) Size-Controlled Model Co Nanoparticle Catalysts for $\mathrm{CO}_{2}$ Hydrogenation: Synthesis, Characterization, and Catalytic Reactions. Nano Lett 12:3091-3096

23. Carenco S, Wu C-H, Shavorskiy A, Alayoglu S, Somorjai GA, Bluhm H, Salmeron M (2015) Synthesis and Structural Evolution of Nickel-Cobalt Nanoparticles Under $\mathrm{H}_{2}$ and $\mathrm{CO}_{2}$. Small 11:3045-3053

24. Sharafutdinov I, Elkjaer CF, de Carvalho HWP, Gardini D, Chiarello GL, Damsgaard CD, Wagner JB, Grunwaldt J-D, Dahl S, Chorkendorff I (2014) Intermetallic compounds of Ni and $\mathrm{Ga}$ as catalysts for the synthesis of methanol. J Catal 320:77-88

25. Studt F, Sharafutdinov I, Abild-Pedersen F, Elkjaer CF, Hummelshøj JS, Dahl S, Chorkendorff I, Nørskov JK (2014) Discovery of a Ni-Ga catalyst for carbon dioxide reduction to methanol. Nat Chem 6:320-324

26. Behrens M, Studt F, Kasatkin I, Kühl S, Hävecker M, Abild-Pedersen F, Zander S, Girgsdies F, Kurr P, Kniep B-L, Tovar M, Fischer RW, Nørskov JK, Schlögl R (2012) The Active Site of Methanol Synthesis over $\mathrm{Cu} / \mathrm{ZnO} / \mathrm{Al}_{2} \mathrm{O}_{3}$ Industrial Catalysts. Science 336:893897

27. Graciani J, Mudiyanselage K, Xu F, Baber AE, Evans J, Senanayake SD, Stacchiola DJ, Liu P, Hrbek J, Fernández Sanz J, Rodriguez JA (2014) Highly active copper-ceria and copperceria-titania catalysts for methanol synthesis from $\mathrm{CO}_{2}$. Science 345:546-550

28. Fiordaliso EM, Sharafutdinov I, Carvalho HWP, Grunwaldt J-D, Hansen TW, Chorkendorff I, Wagner JB, Damsgaard CD (2015) Intermetallic $\mathrm{GaPd}_{2}$ Nanoparticles on $\mathrm{SiO}_{2}$ for Low-Pressure $\mathrm{CO}_{2}$ Hydrogenation to Methanol: Catalytic Performance and In Situ Characterization. ACS Catal 5:5827-5836

29. Hong J, Zhang W, Ren J, Xu R (2013) Photocatalytic reduction of $\mathrm{CO}_{2}$ : a brief review on product analysis and systematic methods. Anal Methods 5:1086-1097

30. Yang C-C, Yu Y-H, van der Linden B, Wu JCS, Mul G (2010) Artificial Photosynthesis over Crystalline $\mathrm{TiO}_{2}$-Based Catalysts: Fact or Fiction? J Am Chem Soc 132:8398-8406

31. Kohno Y, Tanaka T, Funabiki T, Yoshida S (1998) Identification and reactivity of a surface intermediate in the photoreduction of $\mathrm{CO}_{2}$ with $\mathrm{H}_{2}$ over $\mathrm{ZrO}_{2}$. J Chem Soc Faraday Trans 94:1875-1880

32. Kohno Y, Tanaka T, Funabiki T, Yoshida S (1997) Photoreduction of carbon dioxide with hydrogen over $\mathrm{ZrO}_{2}$. Chem Commun 841-842

33. Kohno Y, Tanaka T, Funabiki T, Yoshida S (2000) Photoreduction of $\mathrm{CO}_{2}$ with $\mathrm{H}_{2}$ over $\mathrm{ZrO}_{2}$. A study of interaction of hydrogen with photoexcited $\mathrm{CO}_{2}$. Phys Chem Chem Phys 2:2635-2639 
34. Kohno Y, Ishikawa H, Tanaka T, Funabiki T, Yoshida S (2001) Photoreduction of carbon dioxide by hydrogen over magnesium oxide. Phys Chem Chem Phys 3:1108-1113

35. Teramura K, Tanaka T, Ishikawa H, Kohno Y, Funabiki T (2004) Photocatalytic reduction of $\mathrm{CO}_{2}$ to $\mathrm{CO}$ in the presence of $\mathrm{H}_{2}$ or $\mathrm{CH}_{4}$ as a reductant over MgO. J Phys Chem B 108:346-354

36. Teramura K, Okuoka S-i, Tsuneoka H, Shishido T, Tanaka T (2010) Photocatalytic reduction of $\mathrm{CO}_{2}$ using $\mathrm{H}_{2}$ as reductant over $\mathrm{ATaO}_{3}$ photocatalysts $(\mathrm{A}=\mathrm{Li}, \mathrm{Na}, \mathrm{K})$. Appl Catal B-Environ 96:565-568

37. Teramura K, Tsuneoka $\mathrm{H}$, Shishido $\mathrm{T}$, Tanaka $\mathrm{T}$ (2008) Effect of $\mathrm{H}_{2}$ gas as a reductant on photoreduction of $\mathrm{CO}_{2}$ over a $\mathrm{Ga}_{2} \mathrm{O}_{3}$ photocatalyst. Chemical Physics Letters 467:191-194

38. Tsuneoka H, Teramura K, Shishido T, Tanaka T (2010) Adsorbed Species of $\mathrm{CO}_{2}$ and $\mathrm{H}_{2}$ on $\mathrm{Ga}_{2} \mathrm{O}_{3}$ for the Photocatalytic Reduction of $\mathrm{CO}_{2}$. J Phys Chem C 114:8892-8898

39. Kohno Y, Hayashi H, Takenaka S, Tanaka T, Funabiki T, Yoshida S (1999) Photoenhanced reduction of carbon dioxide with hydrogen over $\mathrm{Rh} / \mathrm{TiO}_{2}$. J Photochem Photobiol AChem 126:117-123

40. Lo C-C, Hung C-H, Yuan C-S, Wu J-F (2007) Photoreduction of carbon dioxide with $\mathrm{H}_{2}$ and $\mathrm{H}_{2} \mathrm{O}$ over $\mathrm{TiO}_{2}$ and $\mathrm{ZrO}_{2}$ in a circulated photocatalytic reactor. Sol Energy Mater Sol Cells 91:1765-1774

41. Hoch LB, Wood TE, O'Brien PG, Liao K, Reyes LM, Mims CA, Ozin GA (2014) The Rational Design of a Single-Component Photocatalyst for Gas-Phase $\mathrm{CO}_{2}$ Reduction Using Both UV and Visible Light. Adv Sci 1:1400013

42. Li M, Li P, Chang K, Wang T, Liu L, Kang Q, Ouyang S, Ye J (2015) Highly efficient and stable photocatalytic reduction of $\mathrm{CO}_{2}$ to $\mathrm{CH}_{4}$ over $\mathrm{Ru}$ loaded $\mathrm{NaTaO}_{3}$. Chem Commun 51:7645-7648

43. Tahir M, Amin NS (2015) Photocatalytic $\mathrm{CO}_{2}$ reduction with $\mathrm{H}_{2}$ as reductant over copper and indium co-doped $\mathrm{TiO}_{2}$ nanocatalysts in a monolith photoreactor. Appl Catal A-Gen 493:90-102

44. Tahir M, Amin NS (2016) Performance analysis of nanostructured $\mathrm{NiO}-\mathrm{In}_{2} \mathrm{O}_{3} / \mathrm{TiO}_{2}$ catalyst for $\mathrm{CO}_{2}$ photoreduction with $\mathrm{H}_{2}$ in a monolith photoreactor. Chem Eng J 285:635-649

45. Ahmed N, Shibata Y, Taniguchi T, Izumi Y (2011) Photocatalytic conversion of carbon dioxide into methanol using zinc-copper-M(III) ( $\mathrm{M}=$ aluminum, gallium) layered double hydroxides. J Catal 279:123-135

46. Ahmed N, Morikawa M, Izumi Y (2012) Photocatalytic conversion of carbon dioxide into methanol using optimized layered double hydroxide catalysts. Catal Today 185:263-269

47. Yang C-C, Vernimmen J, Meynen V, Cool P, Mul G (2011) Mechanistic study of hydrocarbon formation in photocatalytic $\mathrm{CO}_{2}$ reduction over Ti-SBA-15. J Catal 284:1-8

48. Thampi KR, Kiwi J, Grätzel M (1987) Methanation and photo-methanation of carbondioxide at room-temperature and atmospheric pressure. Nature 327:506-508

49. O'Brien PG, Sandhel A, Wood TE, Jelle AA, Hoch LB, Perovic DD, Mims CA, Ozin GA (2014) Photomethanation of Gaseous $\mathrm{CO}_{2}$ over Ru/Silicon Nanowire Catalysts with Visible and Near-Infrared Photons. Adv Sci 1:1400001

50. Meng X, Wang T, Liu L, Ouyang S, Li P, Hu H, Kako T, Iwai H, Tanaka A, Ye J (2014) Photothermal Conversion of $\mathrm{CO}_{2}$ into $\mathrm{CH}_{4}$ with $\mathrm{H}_{2}$ over Group VIII Nanocatalysts: An Alternative Approach for Solar Fuel Production. Angew Chem-Int Ed 53:11478-11482

51. Sastre F, Puga AV, Liu L, Corma A, García H (2014) Complete Photocatalytic Reduction of $\mathrm{CO}_{2}$ to Methane by $\mathrm{H}_{2}$ under Solar Light Irradiation. J Am Chem Soc 136:67986801

52. Morikawa M, Ahmed N, Yoshida Y, Izumi Y (2014) Photoconversion of carbon dioxide in zinc-copper-gallium layered double hydroxides: The kinetics to hydrogen carbonate and further to CO/methanol. Appl Catal B-Environ 144:561-569

53. Sabatier P (1910) Making methane or mixtures of methane and hydrogen, US Pat. 956734

54. Melsheimer J, Guo W, Ziegler D, Wesemann M, Schlögl R (1991) Methanation of carbon dioxide over Ru/Titania at room temperature: explorations for a photoassisted catalytic reaction. Catal Lett 11:157-168 
55. Lin X, Yang K, Si R, Chen X, Dai W, Fu X (2014) Photoassisted catalytic methanation of $\mathrm{CO}$ in $\mathrm{H}_{2}$-rich stream over $\mathrm{Ru} / \mathrm{TiO}_{2}$. Appl Catal B-Environ 147:585-591

56. Lin X, Lin L, Huang K, Chen X, Dai W, Fu X (2015) CO methanation promoted by UV irradiation over $\mathrm{Ni} / \mathrm{TiO}_{2}$. Appl Catal B-Environ 168-169:416-422

57. Sastre F, Oteri M, Corma A, García H (2013) Photocatalytic water gas shift using visible or simulated solar light for the efficient, room-temperature hydrogen generation. Energy Environ Sci 6:2211-2215

58. Sastre F, Corma A, García H (2013) Visible-Light Photocatalytic Conversion of Carbon Monoxide to Methane by Nickel(II) Oxide. Angew Chem-Int Ed 52:12983-12987 